\title{
ORLICZ SPACES ISOMORPHIC TO STRICTLY CONVEX SPACES ${ }^{1}$
}

\section{K. SUNDARESAN}

In this paper it is proved that if $(X, S, \mu)$ is a measure space then the Orlicz space $L_{\Phi}(X, S, \mu)$ is isomorphic to a strictly convex Banach space if the Young function $\Phi$ satisfies the growth condition $G_{1}$ or $G_{2}$ defined below according as $\mu(X)$ is infinite or $\mu(X)$ is finite. As corollaries of this theorem we obtain (1) a simple proof of the known theorem, Day [2], that every $L$-space is isomorphic to a strictly convex space, and (2) every reflexive Orlicz space is isomorphic to a strictly convex space if $(X, S, \mu)$ is $\sigma$-finite.

Definition 1. If $\Phi$ is a Young function and $(X, S, \mu)$ is a measure space then $L_{\Phi}(X, S, \mu)$ is the linear space of all complex valued $\mu$ measurable functions $f$ such that $\Phi(|k f|)$ is $\mu$-summable for some nonzero real number $k$ after the usual identification that $f=g$ if and only if $f=g$ a.e.

It is known (Weiss [5]) that $L_{\Phi}(X, S, \mu)$ is a Banach space under the norm \|\|$_{\Phi}$ defined by

$$
\|f\|_{\Phi}=\inf \left\{1 / a \mid a \geqq 0, \int_{X} \Phi(|a f|) d \mu \leqq 1\right\} .
$$

In what follows we assume $\Phi$ is a nonzero Young function and $L_{\Phi}$ is the Banach space $\left(L_{\Phi}(X, S, \mu),\|\|_{\Phi}\right)$.

Definition 2. The Young function $\Phi$ is said to satisfy the condition $G_{1}$ if there exists a constant $C$ such that $\Phi(2 u) \leqq C \Phi(u)$ for all $u \geqq 0$, and $\Phi$ is said to satisfy $G_{2}$ if the above inequality is true for large $u$.

REMARK 1. If $\mu(X)$ is infinite and $\Phi$ satisfies the condition $G_{1}$ then the integral $M(f)=\int_{X} \Phi(|f|) d \mu$ is finite for all $f$ in $L_{\Phi}$. If $\mu(X)$ is finite then the same is true if $\Phi$ satisfies the condition $G_{2}$. Further it is verified that if $M(f)$ is finite for all $f$ in $L_{\Phi}$ then $\|f\|_{\Phi}=1$ if and only if $M(f)=1$.

We establish two lemmas before proceeding to the main theorem.

Lemma 1. If $\Phi$ is a Young function then there exists a Young function $\Phi_{1}$ such that (i) $\Phi(u) \leqq \Phi_{1}(u) \leqq 2 \Phi(u)$ and (ii) $\Phi_{1}(u)$ is strictly convex in $] v, \infty\left[\right.$ where $v=\sup \Phi^{-1}(0)$.

Received by the editors December 3, 1965.

1 This work was supported in part by ONR Grant 1-51021. 
Proof. It suffices to construct a Young function $\Phi_{1}$ satisfying the inequality (i) in the lemma with its left derivative strictly increasing in the interval $] v, \infty[$.

First we note that since $\Phi$ is a Young function, the left derivative of $\Phi$, say, $\phi$ exists at all points in $] 0, \infty[$ and further $\Phi$ admits the integral representation $\Phi(u)=\int_{[0, u]} \phi d \lambda$ where $\lambda$ is the Lebesgue measure.

Let $\phi_{0}(t)$ be the real valued function defined on $[0, \infty$ [ by setting

$$
\phi_{0}(t)=\frac{t}{2} \phi(t)
$$

if $0 \leqq t \leqq 1$, and

$$
\phi_{0}(t)=\left(\sum_{i=1}^{n-1} \frac{1}{2^{2}} \phi(i)\right)+\frac{t-(n-1)}{2^{n}} \phi(t)
$$

if $n-1 \leqq t \leqq n$ where the integer $n \geqq 2$. Since $\phi$ is nondecreasing, $\phi_{0}$ is nondecreasing and $\phi_{0}(t) \leqq \phi(t)$ for $t \geqq 0$. Further (a) $\phi_{0}(t)=0$ if and only if $\phi(t)=0$ and (b) $\phi_{0}\left(t_{1}\right) \neq 0 \neq \phi_{0}\left(t_{2}\right)$ and $t_{1}>t_{2}$ imply $\phi_{0}\left(t_{1}\right)>\phi_{0}\left(t_{2}\right)$. Now consider the Young function defined by

$$
\Phi_{0}(u)=\int_{[0, u]} \phi_{0}(t) d \lambda .
$$

It follows from (a) above that $\Phi_{0}(u)=0$ if and only if $\Phi(u)=0$. Hence from (b) it follows that $\phi_{0}$ is strictly increasing in the interval ]v, $\infty[$. Thus $\Phi_{0}$ is strictly convex in $] v, \infty\left[\right.$. Now the Young function $\Phi_{1}=\Phi$ $+\Phi_{0}$ has the desired properties.

LEMmA 2. If the Young function $\Phi$ satisfies the condition $G_{2}$ and $\mu(X)$ is finite then there exists a Young function $\Phi^{\prime}$ such that $\Phi^{\prime}(u)>0$ for all $u>0$ and $L_{\Phi}$ is isomorphic to $L_{\Phi^{\prime}}$.

Proof. Since $\Phi$ satisfies the condition $G_{2}$ there exist two nonnegative real numbers $C$ and $u_{0}$ such that for all $u \geqq u_{0}, \Phi(2 u) \leqq C \Phi(u)$. Further since $\Phi$ is nonzero, $C \geqq 2$. Let $\Phi^{\prime}$ be the function on $[0, \infty[$ defined by setting

$$
\Phi^{\prime}(u)=\sum_{n \geq 0} \frac{1}{C^{2 n}} \Phi\left(2^{n} u\right) .
$$

We prove first that $\Phi^{\prime}(u)<\infty$ for all $u \geqq 0$. Let $u_{1} \geqq 0$. Let $N$ be the smallest in teger $\geqq 0$ such that $2^{N} u_{1} \geqq u_{0}$. Then it is verified that 


$$
\begin{aligned}
\Phi^{\prime}\left(u_{1}\right) & =\sum_{n<N} \frac{1}{C^{2 n}} \Phi\left(2^{n} u_{1}\right)+\sum_{n \geqq N} \frac{1}{C^{2 n}} \Phi\left(2^{n} u_{1}\right) \\
& \leqq \sum_{n<N} \frac{1}{C^{2 n}} \Phi\left(2^{n} u_{1}\right)+\sum \frac{c^{n-N}}{C^{2 n}} \Phi\left(2^{N} u_{1}\right) \\
& \leqq \sum_{n<N} \frac{1}{C^{2 n}} \Phi\left(2^{n} u_{1}\right)+\frac{C}{C-1} \Phi\left(2^{n} u_{1}\right)
\end{aligned}
$$

Hence $\Phi^{\prime}\left(u_{1}\right)$ is finite. Thus $\Phi^{\prime}(u)$ is a convex real valued function defined on $\left[0, \infty\left[\right.\right.$ with $\Phi^{\prime}(0)=0$ and $\Phi^{\prime}$ is a Young function. Next let $f \in L_{\Phi}$. From Remark 1 it follows that $\Phi(|f|)$ is $\mu$-summable and if $E_{1}=\left\{x|| f(x) \mid \geqq u_{0}\right\}$ and $E_{2}=X \sim E_{1}$, then

$$
\int_{\mathbf{X}} \Phi^{\prime}(|f|) d \mu \leqq \Phi^{\prime}\left(u_{0}\right) \mu\left(E_{2}\right)+\frac{C}{C-1} \int_{E_{1}} \Phi(|f|) d \mu
$$

since $\Phi(2 u) \leqq C \Phi(u)$ for $u \geqq u_{0}$. Thus $\Phi^{\prime}(|f|)$ is also $\mu$-summable and $f \in L_{\Phi^{\prime}}$. Thus $L_{\Phi}, L_{\Phi^{\prime}}$ are Banach spaces consisting of the same functions and $\|f\|_{\Phi} \leqq\|f\|_{\Phi^{\prime}}$. Hence $L_{\Phi}$ is isomorphic to $L_{\Phi^{\prime}}$. Since $\Phi(u)>0$ for large $u, \Phi^{\prime}(u)>0$ for all $u>0$.

REMARK 2. Since $\Phi$ satisfies the condition $G_{2}$ the function $\Phi_{1}$ also satisfies the condition $G_{2}$.

ThEOREM 1. If $\Phi$ satisfies the condition $G_{1}$ then $L_{\Phi}$ is isomorphic to a strictly convex space. If $\mu(X)$ is finite and $\Phi$ satisfies $G_{2}$ then $L_{\Phi}$ is isomorphic to a strictly convex space.

Proof. Let $\Phi$ satisfy the condition $G_{2}$. Hence $\Phi(u)>0$ for all $u>0$. Hence by Lemma 1 there exists a strictly convex Young function $\Phi_{1}$ such that $\Phi(u) \leqq \Phi_{1}(u) \leqq 2 \Phi(u)$. Thus $L_{\Phi}$ and $L_{\Phi_{1}}$ are isomorphic under the identity mapping. Further since $\Phi$ satisfies the condition $G_{1}, \Phi_{1}$ also satisfies the condition $G_{1}$. Thus $\int_{X} \Phi_{1}(|f|) d \mu<\infty$ for all $f \in L_{\Phi_{1}}$. Hence $\|f\|_{\Phi_{1}}=1$ if and only if $\int_{X} \Phi(|f|) d \mu=1$. Thus if $\|f\|_{\Phi_{1}}=\|g\|_{\Phi_{1}}$ $=\|(f+g) / 2\|_{\Phi_{1}}=1$ then

$$
\int_{X}\left[\Phi_{1}\left(\left|\frac{f+g}{2}\right|\right)-\frac{1}{2} \Phi_{1}(|f|)-\frac{1}{2} \Phi_{1}(|g|)\right] d \mu=0 .
$$

Since $\Phi_{1}$ is strictly convex and $\Phi_{1}(u)>0$ for $u>0$ the last equation implies $f=g$ a.e.

Next let $0 \leqq \mu(X)<\infty$ and $\Phi$ satisfy the condition $G_{2}$. Then by Lemma 2 there exists an Young function $\Phi_{1}$ such that $L_{\Phi}$ is isomorphic 
with $L_{\Phi_{1}}$ where $\Phi_{1}(u)>0$ for all $u>0$. Now the first part of Theorem 1 applies to $L_{\Phi_{1}}$ completing the proof of the second part.

Since the above theorem is applicable when $\Phi(u)=u$ the following corollary is obtained.

CoRollary 1 [DAy]. Every L-space is isomorphic to a strictly convex space.

It is not known, Day [3], whether every reflexive Banach space is isomorphic to a strictly convex space. The following corollary settles the problem for Orlicz spaces when the underlying measure space is $\sigma$-finite.

Corollary 2. If $(X, S, \mu)$ is $\sigma$-finite and $L_{\Phi}$ is a reflexive space then $L_{\Phi}$ is isomorphic to a strictly convex space.

Proof. It follows from Theorems 5 and 6 on pages 60 and 61 of Luxemburg [4], that $L_{\Phi}$ is reflexive implies one of the following three statements if $(X, S, \mu)$ is $\sigma$-finite.

(A) $\Phi$ satisfies the condition $G_{1}$.

(B) $0<\mu(X)<\infty$ and $\Phi$ satisfies the condition $G_{2}$.

(C) $L_{\Phi}$ is a separable Banach space.

If we are in the cases (A) and (B) then Theorem 1 is applicable and $L_{\Phi}$ is isomorphic to a strictly convex space. If we are in the case (C) then by a well-known theorem of Clarkson [1], $L_{\Phi}$ is isomorphic to a strictly convex space.

\section{REFERENCES}

1. J. A. Clarkson, Uniformly convex spaces, Trans. Amer. Math. Soc. 40 (1936), 396-414.

2. M. M. Day, Every L-space is isomorphic to a strictly convex space, Proc. Aner. Math. Soc. 8 (1957), 415-417.

3. - Strict convexity and smoothness of normed spaces, Trans. Amer. Math. Soc. 78 (1955), 516-528.

4. W. A. J. Luxemburg, Banach function spaces, Thesis, Technische Hogeschool te Delft, 1955.

5. G. Weiss, A note on Orlicz spaces, Portugal. Math. 15 (1956), 35-47.

Carnegie Institute of Technology 